Anales de Geografía de la Universidad Complutense ISSN: 0211-9803

http://dx.doi.org/10.5209/AGUC.55958

\title{
Cambios en la localización residencial de la inmigración en el área metropolitana de Sevilla: De la preferencia a la restricción residencial ${ }^{1}$
}

\author{
Gwendoline De Oliveira Neves ${ }^{2}$; Claudia Hurtado Rodríguez ${ }^{3}$; Ricardo Iglesias Pascual ${ }^{4}$ \\ Recibido: 8 de julio del 2016 / Enviado a evaluar: 22 de julio del 2016 / Aceptado: 16 de diciembre del 2016
}

Resumen. Los procesos de segregación y preferencia residencial entre la sociedad de acogida y los diferentes grupos étnicos han generado un prolífico debate académico, que se ha plasmado entre otras en las denominadas teorías de la amenaza y el contacto. Este artículo pretende aportar a dicho debate mediante el estudio del comportamiento espacial de la población inmigrante. Para ello se han creado dos grupos de estudio, según su grado de rechazo social en la sociedad española, relacionándolos con la idea de preferencia y restricción residencial. Los resultados en el área metropolitana de Sevilla durante el periodo 2003-2013, muestran una clara separación entre las zonas de concentración residencial de ambos grupos. Dicha división aumenta con el incremento de la inmigración económica en Sevilla, reafirmando la tendencia ya observada en otros estudios a la preferencia de la sociedad de acogida por barrios social y étnicamente uniformes.

Palabras clave: Estrategia residencial; segregación; estigma territorial; downscaling; Sevilla

\section{[en] Changes in the residential location of immigration in Seville metropolitan area: From preference to residential restriction}

Abstract. The processes of segregation and residential preference between the host society and different ethnic groups have produced a productive academic debate which it has been reflected among others in the so-called theories of threat and contact. This paper aims to contribute to this discussion by studying the spatial behavior of the immigrant population. For this purpose have been created two study groups according to their degree of rejection in Spanish society. The results in the metropolitan area of Seville during the period 2003-2013 show a clear separation between residential concentration's areas of both groups. This division increases with the increase of economic immigration in Seville, reaffirming the

1 Este artículo se enmarca dentro del Proyecto de Excelencia del Plan Nacional de I+D+I "Dinámicas y transformaciones territoriales, funcionales y sociales de las áreas metropolitanas españolas en un horizonte de sostenibilidad" (CSO2014-55780-C3-1-P) financiado por el Ministerio de Economía y Competitividad.

2 Universidad Pablo de Olavide

E-mail: gsaroli@upo.es

3 Universidad Pablo de Olavide

E-mail: churrod@upo.es

4 Universidad Pablo de Olavide

E-mail: riglpas@upo.es 
already noted trend in other research to the preference of the host society by social and ethnically uniform neighborhoods.

Keywords: Residential strategy; segregation; territorial stigma; downscaling; Seville.

[fr] Evolution de la situation résidentiel de l'immigration dans la région métropolitaine de Séville: Preference pour restriction résidentiel

Résumé. Les processus de ségrégation et préférence résidentielle entre les sociétés d'accueils et les différents groupes ethniques sont au coeur des débats scientifiques donnant lieu, entre autres, aux théories de la peur et du contact. Cet article prétend enrichir ce débat en proposant une étude des comportements spatiaux de la population immigrante en utilisant deux groupes selon le degrés d'exclusion dans la société espagnole tout en considérant que le comportement de la population la mieux intégrée est représentatif des préférences résidentielles de la population d'accueil. Les résultats analysés entre 2003-2013 au sein de l'aire métropolitaine de Séville illustrent une nette séparation entre les zones de concentrations résidentielles des deux groupes. Cette division, s'accroit à Séville, à mesure que l'immigration économique augmente réaffirmant ainsi la tendance des société d'accueil à préférer les quartiers socialement et ethniquement homogènes.

Mots clés: Stratégies résidentielles; ségrégation; stigmatisation territoriale; downscalling; Séville.

Cómo citar. De Oliveira Neves, G., Hurtado Rodríguez, C. y Iglesias Pascual, R. (2017): Cambios en la localización residencial de la inmigración en el área metropolitana de Sevilla: De la preferencia a la restricción residencial. Anales de Geografía de la Universidad Complutense, 37(1), 107-133.

Sumario. 1. Introducción. 2. Antecedentes teçoricos y sus implicaciones en la investigación. 3. Metodología. 4. Estrategia residenciales, desde la preferencia a la restricción residencial.

5. Concentración y estigmatización territorial de los barrios inmigrantes. 6. Conclusiones. 7. Bibliografía.

\section{Introducción}

A la hora de analizar la segregación residencial que experimentan los diferentes grupos étnicos se pueden encontrar diferentes enfoques académicos que profundizan tanto en los factores que generan dicha segregación como en los elementos subyacentes en la elección del barrio de residencia. A su vez se suele analizar el interés por residir en ámbitos con mayor o menor grado de diversidad etnocultural, los mecanismos que intervienen en la integración o aislamiento de la población inmigrante, así como la tendencia a la autosegregación de los diferentes grupos. Del mismo modo destacan aquellos enfoques que abordan variables relacionadas con la xenofobia, las preferencias residenciales y los estereotipos étnicos.

Sin embargo, metodológicamente en algunos de estos estudios se ha puesto de manifiesto como la utilización de encuesta plantea una cierta limitación en la validez de los resultados ya que los entrevistados, especialmente aquellos con mayor nivel educativo, suelen matizar o encubrir sus opiniones reales en busca de una mayor corrección política de sus opiniones (Krysan, 1998; Cea D'Ancona, 2009). Incluso se ha demostrado como la escala territorial influye en los resultados obtenidos a la hora 
de abordar la preferencia o rechazo por vecindarios con mayor o menor grado de diversidad étnica (Bader y Krysan, 2015). Por otro lado aspectos como el papel de las percepciones que se generan sobre los barrios, las imposiciones del mercado residencial, así como los estereotipos territoriales existentes sobre los ámbitos que albergan mayoritariamente inmigrantes, son igualmente complicados de recoger mediante encuestas pero resultan muy necesarios para conseguir una visión holística de los elementos que explican la segregación (Van der Laan Bouma-Doff, 2007; Crowder y Krysan, 2016; Iglesias Pascual, 2016).

En base a ello el presente artículo propone un análisis del proceso de segregación, concentración y localización residencial de la inmigración en el área metropolitana de Sevilla a través del estudio del comportamiento espacial de la población nacida en el extranjero y su nivel de rechazo en la sociedad de acogida. Con ello se propone desarrollar una visión complementaria a la que ofrecen los estudios basados en registros estadísticos generados a partir de las opiniones de entrevistados, valorando qué aspectos de dichos estudios se pueden comprobar o desestimar a partir del análisis espacial. De este modo se busca superar las divergencias que suelen darse entre las opiniones de los entrevistados sobre sus preferencias residenciales, lo explícito, y lo que realmente hacen a la hora de buscar vivienda, el comportamiento espacial implícito (Havakes, Bader y Krysan, 2016).

Al basar la investigación en el comportamiento espacial y por tanto en el comportamiento implícito y real de los individuos, se ha diferenciado entre la idea de preferencia residencial, aplicándola a aquella población inmigrante que debido a su mayor nivel de ingresos puede realmente elegir dónde residir en el espacio metropolitano y la de restricción, referida a la población procedente de países de menor renta, para los que los condicionantes socioeconómicos y los prejuicios del mercado residencial reduce de manera significativa su capacidad de elección de vecindario.

En este sentido la presente investigación, a partir de las conclusiones alcanzadas en otros estudios (Allport, 1954; Shapiro y Neuberg, 2008; Ibraimovic y Masiero, 2014; Iglesias Pascual, 2016) analiza el comportamiento residencial de la población inmigrante procedente de países con mayor renta y su relación con las preferencias de la sociedad de acogida. Con ello se busca comprobar si la actitud espacial de la sociedad receptora hacia los inmigrantes de menos recursos y mayor rechazo social incide en su comportamiento residencial. Mediante el estudio de la distribución territorial de ambos grupos se pretende valorar si paralelo al incremento de la población nacida en el extranjero en el espacio metropolitano, se produce un aumento de la integración y por tanto un incremento en los ámbitos multiculturales, o si por el contrario aumenta la segregación y el aislamiento del inmigrante económico debido al establecimiento de barrios que buscan la uniformidad socioeconómica. Este análisis espacial permite determinar la evolución de lo que se ha determinado como zonas de preferencia y restricción residencial de la inmigración en el espacio metropolitano (De Oliveira Neves, Hurtado Rodríguez, Iglesias Pascual, 2016). 
Para desarrollar todo lo hasta aquí planteado en primer lugar se ha realizado un acercamiento a los diferentes estudios que analizan los comportamientos que intervienen en la segregación y su continuidad reflexionando sobre qué aspectos se reflejan especialmente en la presente investigación. Posteriormente se explicitan los principios metodológicos, haciendo un especial énfasis en los métodos de desagregación espacial mediante downscaling. Tras el repaso a los principios instrumentales que sostienen la investigación, se analizan las diferentes estrategias residenciales que desarrollan los dos grupos de estudios establecidos para posteriormente, determinar la relación de dichas estrategias con el desarrollo de procesos de estigmatización residencial en el área metropolitana de Sevilla durante el periodo 2003-2013. Por último se reflexiona sobre las posibles aportaciones del análisis espacial desarrollado al debate sobre los factores y causas que se encuentran especialmente en la base de la segregación de la población inmigrante con mayor nivel de rechazo social y menor nivel de ingresos.

\section{Antecedentes teóricos y sus implicaciones en la investigación}

Dentro del estudio de la segregación residencial existen tres grandes temáticas articuladoras del debate académico, que a su vez muestran un importante interés para entender los planteamientos de la presente investigación: las causas que originan la segregación; la tendencia a la autosegregación de los diferentes grupos étnicos y por último las consecuencias del contacto entre grupos de diferentes características etnocultorales y socioeconómicas. A su vez debido, al enfoque aquí propuesto, posteriormente se analiza si las pautas de localización residencial de los extranjeros con mayor nivel de ingresos y aceptación social, coinciden con el comportamiento residencial y actitudinal de la sociedad de acogida hacia los inmigrantes con mayor rechazo social procedentes de países de menor renta, la inmigración económica.

A la hora de analizar los elementos que se encuentran en el origen de la segregación cabe destacar tres grandes interpretaciones para su explicación (Quillian, 2002; Charles, 2003): En primer lugar aquellas que consideran las desigualdades económicas, educacionales y culturales existentes entre los diferentes grupos étnicos y la sociedad de acogida, como las causantes del desigual acceso a los vecindarios debido al menor poder adquisitivo (Crowder et al., 2006). En segundo lugar destacan las investigaciones focalizadas en los prejuicios étnicos a la hora de explicar la elección de vecindario, ya sea por el rechazo a ciertos grupos o nacionalidades o por la preferencia a residir en barrios donde predomine el propio grupo étnico o la sociedad de acogida (Clark, 2009; Krysan et al., 2009). Por último destacan los enfoques centrados en la discriminación que se ejerce desde el mercado residencial a través de los propietarios, arrendadores, agencias inmobiliarias y las trabas a la concesión de hipotecas para explicar la segregación y su continuidad en el tiempo (Van der Laan Bouma-Doff, 2007). El problema de toda esta literatura académica es la manera excluyente en la que plantean sus diferentes interpretaciones, reduciendo 
sus posibilidades de analizar globalmente la segregación (Crowder y Krysan, 2016:19).

Relacionado con aquellas investigaciones que explican las causas de la segregación, el estudio sobre la tendencia a la autosegregación es otra de las grandes temáticas abordadas. En esta línea, en primer lugar se encuentran aquellos trabajos que la entienden como una elección libre por parte de los diferentes grupos. En este caso se debe diferenciar entre la autosegregación de la población de acogida, que tradicionalmente en los estudios anglosajones se relaciona con la población blanca, y la de los grupos étnicos minoritarios. La sociedad de acogida suele preferir vecindarios donde predomine la población de sus mismas características (Clark, 2002, 2009). De este modo la presencia de otros grupos étnicos determina en gran medida las decisiones residenciales de la sociedad de acogida (Clark, 2002:242) e interviene en gran manera en los proceso de filtrado residencial, white flight (Clark, 2009).

Por otro lado sobre la autosegregación de los grupos étnicos minoritarios se pueden encontrar dos enfoques diferenciados. Aquellos que consideran que al igual que la población de acogida, su tendencia a residir en vecindarios con miembros de una misma nacionalidad se debe a una decisión voluntaria para estar más cerca de sus iguales y así beneficiarse de las redes sociales y familiares (Ihlanfeldt y Scafidi 2002; Clark, 2009). Sin embargo se pueden encontrar estudios que sostienen que la autosegregación es consecuencia directa del rechazo y la discriminación que los grupos minoritarios sufren por parte del mercedo residencial y la sociedad de acogida, convirtiéndose estos barrios en zonas de seguridad ante dicha hostilidad social (Charles, 2000; Van der Laan Bouma-Doff, 2007).

En el caso europeo hay investigaciones que destacan este papel de la discriminación y relativizan la idea de autosegregación de los grupos minoritarios al señalar como esta tendencia es mayor entre la sociedad de acogida y los inmigrantes provenientes de países de alta renta que entre la inmigración económica la cual prefiere mayoritariamente vecindarios mixtos. Por tanto esta divergencia entre los deseos por vecindarios mixtos de los inmigrantes provenientes de países con menor renta y su concentración real en lo que se denomina como barrios de inmigrantes, solo puede ser explicado por el papel de la discriminación de la sociedad de acogida (Ibraimovic y Masiero, 2014:706). En este sentido los resultados de la presente investigación muestran como este último aspecto, en combinación con las percepciones e imaginarios sociales que se originan sobre los barrios que tienen una alta presencia de inmigrantes económicos, se ha plasmado en una progresiva concentración en ámbitos barriales muy concretos del espacio metropolitano de Sevilla.

Esto da pie a abordar el tercer gran eje teórico que se ha desarrollado sobre la segregación, el efecto del contacto de la sociedad de acogida con otros grupos minoritarios, especialmente cuando las diferencias entre dicho grupos y la población autóctona son amplias desde el punto de vista socioeconómico, étnico y cultural. El efecto de la llegada de la población foránea y su concentración dentro de la ciudad en las sociedades de acogida es uno de los aspectos epistemológicos que ha generado un 
mayor debate a la hora de abordar el estudio de la segregación y la preferencial residencial. A grandes rasgos se pueden diferenciar entre aquellas investigaciones que centradas en analizar la relación entre el aumento de población extranjera y la aparición de actitudes de miedo y rechazo hacia dicha población, lo que se ha denominado como la teoría de la amenaza (Semionov, Gikman y Krysan, 2007), y la llamada teoría del contacto (Kaufmann y Harris, 2015), para la que la mayor presencia de población extranjera en una comunidad aumenta las posibilidades de contacto con la población autóctona, favorece la reducción de los prejuicios hacia esta población y mejoraría la convivencia a largo plazo (Ihlanfeldt \& Scafidi, 2004: 355; Dewaard, 2015). Otros puntos de vista relativizan la importancia del porcentaje de extranjeros a la hora de la construcción de las percepciones sobre la inmigración, considerando que tiene una mayor incidencia en la sociedad receptora, la rapidez del cambio poblacional y las características étnicas de la nueva población (Newman y Velez, 2014).

En el caso español diferentes investigaciones desarrolladas para ciudades como Madrid, Barcelona, Alicante, Valencia y Almería ${ }^{5}$, consideran que los prejuicios y actitudes de rechazo devienen generalmente en el desarrollo de posturas xenófobas y discriminatorias hacia dicha población (Farley et al., 1994; Herranz de Rafael et al., 2012; Iglesias Pascual, 2015). De hecho los barrios donde se concentran la población inmigrante, suelen verse estigmatizados al relacionarse por parte de la sociedad de acogida como espacios problemáticos, relacionados con estereotipos negativos (Charles, 2000; De Souza Briggs, 2005; Iglesias Pascual, 2016), influyendo directamente en la manera en la que los vecindarios son percibidos y determinando la búsqueda de vivienda (Charles 2001). A su vez estos estereotipos se ven especialmente determinados por la nacionalidad o las características del grupo minoritario en cuestión, ya que tanto en el ámbito norteamericano (Bader y Krysan, 2015), como en el europeo (Van der Laan Bouma-Doff, 2007; Pettigrew, Wagner, Crist, 2010; Iglesias Pascual, 2016), el origen del foráneo determina la manera en que se percibe el barrio desde la sociedad de acogida.

Por tanto, aspectos como las relaciones históricas con los países de procedencia, las diferencias raciales, religiosas y especialmente socioeconómicas determinan en gran medida el desarrollo de actitudes de rechazo hacia el extranjero. En este sentido en el caso español, tanto los diferentes estudios OPIA realizados desde Andalucía como por los informes de OBERAXE sobre la evolución del racismo y la xenofobia (Cea D'Ancona y Vallés, 2015), muestran que las nacionalidades con mayor rechazo social entre la población española son aquellas procedentes del Magreb, árabes, miembros de los países del Este de Europa, especialmente la rumana, y latinoamericanos.

\footnotetext{
${ }^{5}$ En dichas investigaciones se establece entre el $15 \%$ y el $20 \%$ de población extranjera el umbral en el que comienzan a aparecer actitudes de miedo y rechazo hacia el extranjero en un ámbito territorial.
} 
Atendiendo a la importancia de estas percepciones y estereotipos en España y Andalucía, en la presente investigación se ha determinado utilizar el país de nacimiento por encima del hecho de ser población nacionalizada o extranjera a la hora de seleccionar los datos. Es decir se ha priorizado el origen etnocultural sobre la situación administrativa, ya que es este primer aspecto, la clave a la hora de entender las percepciones y actitudes de rechazo que se construyen socialmente desde la población de acogida. Actitudes que suelen plasmarse en la generalización del rechazo para acceder al mercado de vivienda de propiedad y alquiler a la población perteneciente a las nacionalidades más estigmatizadas por la población autóctona (Iglesias Pascual, 2015, 2016; Van der Bracht, Coenen y Van de Putte, 2015). Dichas actitudes de rechazo hacia estas nacionalidades juegan un papel crucial en los procesos de filtrado y segregación residencial de la población extranjera y ponen en duda los principios defendidos por las investigaciones agrupadas dentro de las teorías del contacto, aspecto que de manera general se ve confirmado por los resultados aquí presentados.

Por tanto, para poder analizar todas estas cuestiones, en la investigación se han establecidos dos grandes grupos de estudio: en un primer grupo (Grupo 1) se han agrupado aquellas nacionalidades que no presentan nivel de rechazo por la población española (norteamericanos y miembros de la UE, excepto polacos, búlgaros y rumanos). Un segundo grupo (Grupo 2), engloba aquellas nacionalidades que presentan mayor nivel de rechazo social por parte de la sociedad de acogida (centroamericanos, sudamericanos, asiáticos, africanos, miembros de la Europa no comunitaria junto a polacos, búlgaros y rumanos). Con la formación de estos dos grupos se pretende analizar las pautas residenciales de las diferentes nacionalidades, su evolución territorial a lo largo del tiempo, así como determinar si ambos grupos, conviven o presentan distribuciones residenciales excluyentes en el espacio metropolitano de Sevilla. Dicho ámbito territorial se caracteriza por una escasa presencia de inmigrantes recibidos en un corto espacio de tiempo a partir de 2003, localizados en puntos muy concentrados y concretos del espacio metropolitano (Iglesias Pascual, 2015). De este modo el espacio y el periodo de tiempo seleccionado permite analizar el proceso socioespacial que se genera en una sociedad de acogida ante el inicio de la llegada de población foránea.

A su vez, partiendo de investigaciones que muestran como los miembros de grupos minoritarios, influenciados por las normas sociales del grupo mayoritario, tienen en cuenta dicha norma social en su expresión de prejuicio hacia otras minorías (Allport, 1954; Shapiro y Neuberg, 2008), se ha planteado tomar el comportamiento de los miembros de grupo 1, como reflejo de las actitudes y el comportamiento residencial de la sociedad de acogida. Este aspecto se acentúa aún más cuando el status y las características del grupo minoritario es similar al de la sociedad de acogida lo que genera que no se produzca una identificación de dicho colectivo con el estereotipo negativo de inmigrante (Iglesias Pascual, 2016). De hecho estudios en países europeos (Ibraimovic y Masiero, 2014), reflejan como la ausencia de prejuicios negativos permite a la inmigración procedente de países con mayor renta, asentarse en 
los vecindarios más atractivos de la ciudad desde el punto de vista socioeconómico y ambiental, aquellos en los que predomina la población autóctona. Sin embargo los denominados como inmigrantes económicos, con mayor rechazo social, tienden a residir en los ámbitos metropolitanos menos atractivos y más baratos. Con todo ello se pretende analizar si el comportamiento de ambos grupos plasma en el territorio una jerarquía espacial fruto de la estrategia de los grupos de mayor renta (Pahl, 1975), personalizados en este caso en el grupo 1, para separarse de la población más desfavorecida, representada en el artículo como el grupo 2. Por último a partir de la localización de las principales concentraciones del grupo 2 de población extranjera se aborda la evolución de las zonas de la ciudad estigmatizadas como barrios de inmigrantes por parte de la población autóctona, así como la posible creación de nuevas zonas de estigma y rechazo socioterritorial relacionada con la inmigración.

\section{Metodología}

Desde diversas disciplinas, y en particular desde la Geografía, los estudios donde se plantea una aproximación espacial del territorio están inevitablemente condicionados por un problema ya recurrente relacionado con la imposición de unidades espaciales artificiales para la representación de variables de índole territorial. De modo que la información de partida varía en función del tamaño y distribución de la unidad espacial utilizada en el análisis. Este problema, conocido como "Problema de la Unidad Espacial Modificable" (PUEM), fue planteado por Openshaw (1984) y se ha convertido en una cuestión muy discutida a la hora de identificar la unidad de referencia apropiada para abordar estudios territoriales, especialmente cuando la información se ve limitada y distorsionada por la artificialidad y la heterogeneidad espacial propia de las unidades administrativas. Para poder mitigar los efectos del PUEM, y siguiendo la metodología propuesta por Gallego (2010), Goerlich y Cantarino (2013) y Hurtado Rodríguez y De Oliveira Neves (2015), la aproximación aquí desarrollada plantea en su primera fase la desagregación de la información de partida en unidades espaciales menores y homogéneas utilizando para ello información auxiliar como la cobertura o usos del suelo, de modo que se asocia directamente la información demográfica de partida con aquellas zonas dedicadas a un uso residencial.

Así para una mejor aproximación a la distribución poblacional real, se desagrega la información de ocupación del suelo en base a tipologías morfológicas de usos residenciales que se entienden directamente relacionadas con la densidad poblacional - residencial continuo y discontinuo - transfiriendo así la información de forma no homogénea a lo largo de las unidades de destino. La aplicación de este método de tipo downscaling para la desagregación de la distribución de la población extranjera en el área metropolitana de Sevilla ya ha sido desarrollada en estudios previos (De Oliveira Neves, Hurtado Rodríguez e Iglesias Pascual, 2016), partiendo de la información de la población nacida en el extranjero por secciones censales para el periodo 20032007-2013 y del Mapa de Usos y Coberturas Vegetales de Andalucía a escala 
1:25.000 (MUCVA), disponible para los años 2003 y 2007 y actualizado en este trabajo a 2013 gracias a la identificación de nuevas ocupaciones residenciales con la ayuda de los servicios interoperables (WMS) de la ortofoto de máxima actualidad del Plan Nacional de Ortofotografía Aérea (PNOA) y el Sistema de Información sobre Ocupación del Suelo de España (SIOSE).

Los resultados obtenidos en dicho estudio demuestran la fiabilidad y la rigurosidad de la aproximación y de las fuentes utilizadas en el análisis de entornos metropolitanos, obteniéndose una malla regular o grid de $100 \times 100$ metros con la distribución de la población nacida en el extranjero para los dos grupos definidos en base a su proporción sobre la población total en los dos periodos estudiados, 20032007 y $2007-2013$.

Mediante esta aproximación se procede en un primer momento y con el fin de entender las pautas de localización residencial, a clasificar las zonas de mayor concentración de en base a tres casuísticas; predominancia del grupo 1, predominancia del grupo 2 o predominancia compartida en los casos de coexistencia de ambos grupos en una misma zona. Para ello se toma la distribución de las concentraciones de población extranjera obtenidas en el grid de partida (Tabla 1) y se determina como umbral de clasificación el grado de dispersión de las zonas de mayor concentración para los dos grupos de extranjeros, esto es valores mayores al promedio + desviación típica. De modo que se consideran las siguientes agrupaciones:

Predominancia o preferencia residencial grupo 1 si $C_{\mathrm{g} 1}>\overline{\mathrm{X}}_{\mathrm{g} 1} \pm \sigma_{\mathrm{g} 1} \mathrm{y} \mathrm{C}_{\mathrm{g} 2}<\overline{\mathrm{X}}_{\mathrm{g} 2}+\sigma_{\mathrm{g} 2}$ Predominancia residencial grupo 2 si $_{\mathrm{g} 2}>\overline{\mathrm{x}}_{\mathrm{g} 2} \pm \sigma_{\mathrm{g} 2} \mathrm{y} \mathrm{C} \mathrm{C}_{\mathrm{g} 1}<\overline{\mathrm{x}}_{\mathrm{g} 1} \pm \sigma_{\mathrm{g} 1}$ Predominancia residencial compartida si $\mathrm{C}_{\mathrm{g} 1}>\overline{\mathrm{x}}_{\mathrm{g} 1}+\sigma_{\mathrm{g} 1} \mathrm{y} \mathrm{C} \mathrm{C}_{\mathrm{g} 2}>\overline{\mathrm{x}}_{\mathrm{g} 2}+\sigma_{\mathrm{g} 2}$

Dónde:

$\overline{\mathrm{X}}_{\mathrm{g} 1 \text { y }} \overline{\mathrm{X}}_{\mathrm{g} 2 \text { : }}$ son el promedio según la distribución de los grupos 1 y 2 .

$\sigma_{\mathrm{g} 1}$ y $\sigma_{\mathrm{g} 2}$ son la desviación típica según la distribución de los grupos 1 y 2 .

$\mathrm{C}_{\mathrm{g} 1}$ y $\mathrm{C}_{\mathrm{g} 2}$ s son el valor de la concentración de extranjeros para los grupos 1 y 2 .

Tabla 1. Estadísticos según la distribución de población extranjera (porcentaje extranjeros /población total). 2003, 2007 y 2013.

\begin{tabular}{cccc|ccc} 
& \multicolumn{3}{c|}{ Grupo 1 } & \multicolumn{3}{c}{ Grupo 2 } \\
\cline { 2 - 6 } & $\mathbf{2 0 0 3}$ & $\mathbf{2 0 0 7}$ & $\mathbf{2 0 1 3}$ & $\mathbf{2 0 0 3}$ & $\mathbf{2 0 0 7}$ & $\mathbf{2 0 1 3}$ \\
\hline $\begin{array}{c}\text { Promedio } \\
(\overline{\mathbf{x}})\end{array}$ & 1,28 & 1,55 & 1,89 & 1,60 & 2,53 & 3,98 \\
\hline $\begin{array}{c}\text { Desviación } \\
\text { típica }(\boldsymbol{\sigma})\end{array}$ & 1,04 & 1,08 & 1,49 & 1,20 & 1,75 & 2,73 \\
\hline \begin{tabular}{c}
$\overline{\mathbf{X}}+\mathbf{1 \sigma}$ \\
\cline { 2 - 6 }
\end{tabular} & $\mathbf{2 , 3 2}$ & $\mathbf{2 , 6 3}$ & $\mathbf{3 , 3 8}$ & $\mathbf{2 , 8 0}$ & $\mathbf{4 , 2 8}$ & $\mathbf{6 , 7 1}$ \\
\hline
\end{tabular}

Fuente: Elaboración propia a partir de grid de población. Padrón Municipal de Habitantes 2003, 2007 y 2013 y MUCVA 2003, 2007 y actualización por elaboración propia a 2013 
Finalmente se representa espacialmente la distribución de estas tres agrupaciones en relación a las dos tipologías de morfología urbana residencial seleccionadas. Tipologías que son directamente extraídas de la cobertura de usos del suelo utilizada como información espacial de partida para el diseño del grid de población extranjera; urbano residencial continuo y discontinuo. De este modo se pueden definir patrones específicos en la localización espacial de los grupos de extranjeros en base a la tipología morfológica del tejido residencial y comprobar si la posible segregación residencial o el mismo rechazo social hacia ciertos grupos étnicos está a su vez relacionado con las características morfológicas del tejido urbano donde se localizan estos procesos.

Esta aproximación metodológica (Figura 1) ofrece el desarrollo de una herramienta visual e intuitiva que permite analizar los procesos de evolución de concentración residencial de la población extranjera, pudiéndose relacionar con aquellas zonas más proclives al desarrollo de procesos de estigmatización territorial relacionados con altos porcentajes de inmigrantes.

Figura1. Diagrama de flujo para la aproximación metodológica propuesta.

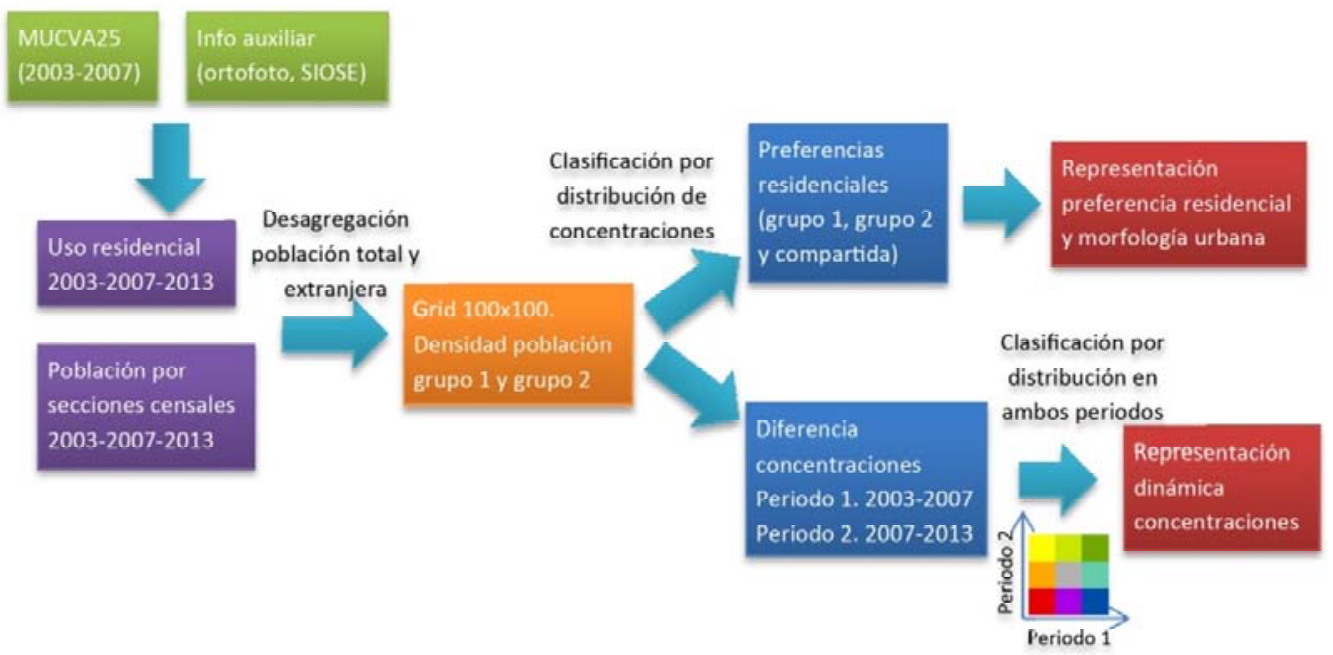

Fuente: Elaboración propia

Una vez analizadas las pautas de localización espacial en 2013, esta aproximación dinámica permite visualizar los procesos de segregación residencial distinguiendo entre los periodos 2003-2007 y 2007-2013. Mediante la representación de las variaciones de concentraciones en un gráfico de doble eje (Figura 2), se puede sintetizar la información de origen gracias a la clasificación de esta variable para ambos periodos. Con el fin de poder visualizar los casos más extremos, se ha tomado 
como umbral para cada periodo o eje, la desviación típica en relación al valor promedio, de modo que podemos definir 3 rangos para su clasificación:

- Variación de concentraciones menores al valor promedio - desviación ( - $1 \sigma)$ $\rightarrow$ Disminución de concentración.

- Variación de concentraciones dentro de la media $\rightarrow$ Variación de concentración dentro de la media.

- Variación de concentraciones mayores al valor medio + desviación $(+1 \sigma) \rightarrow$ Aumento de concentración.

Figura 2. Representación gráfica de doble eje para la clasificación de la evolución de población extranjera en ambos periodos.

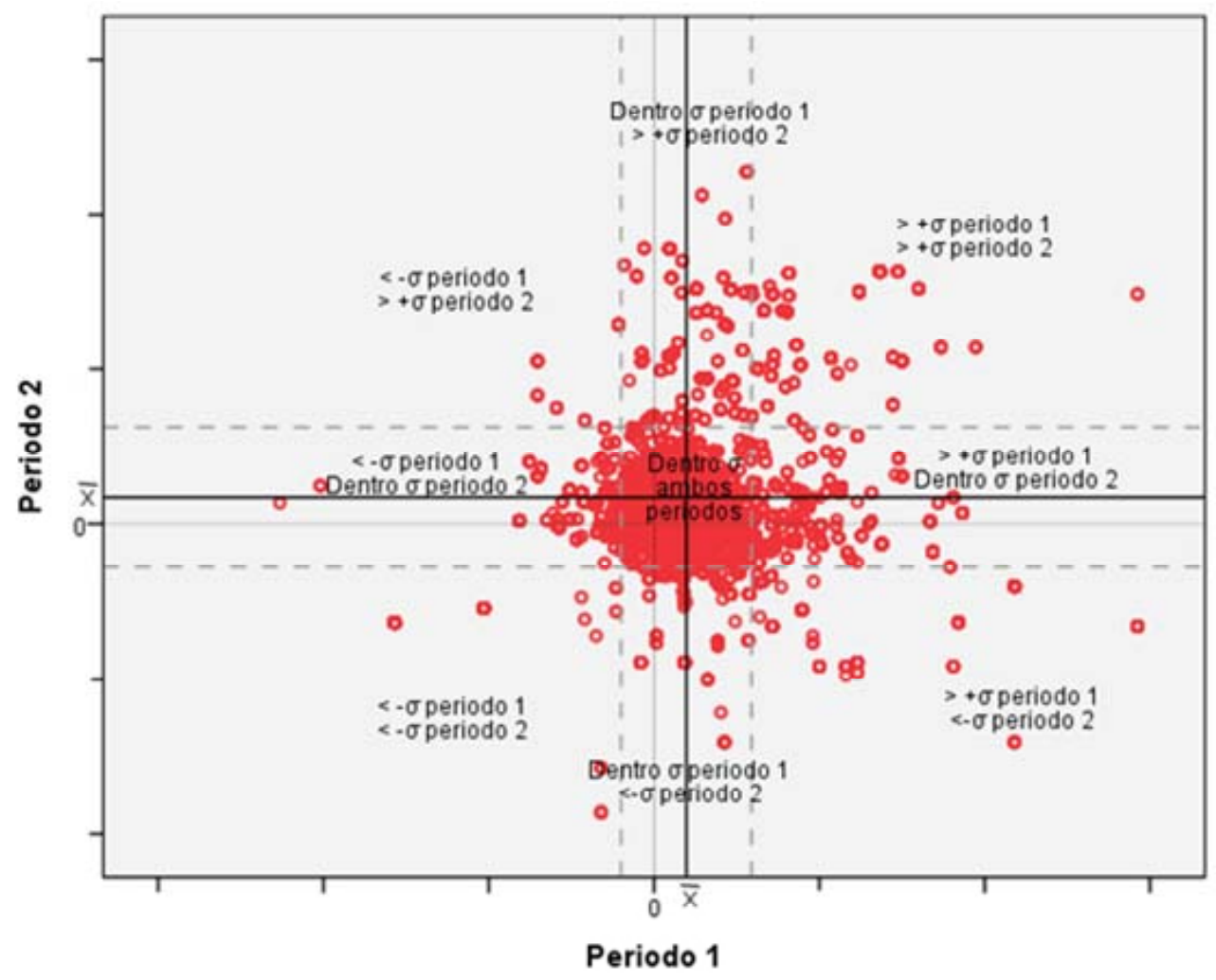

Fuente: Elaboración propia

Para facilitar su lectura se ha sintetizado la información en nueve clases que pueden ser representadas (Figura 3): rojo para las zonas cuya concentración de población extranjera ha disminuido para los dos periodos (zonas más cercanas al 
origen de ambos ejes) y verde para el caso opuesto. Pasando por los casos donde esta concentración ha aumentado en el primer periodo pero disminuye en el segundo (azul) o viceversa (amarillo). Mientras que aquellas zonas cuya concentración se ha mantenido alrededor de la media para ambos periodos son la que quedan en la zona central del gráfico (gris).

Figura 3. Aplicación cromática sobre las clases definidas para la representación cartográfica de la evolución de población nacida en el extranjero.

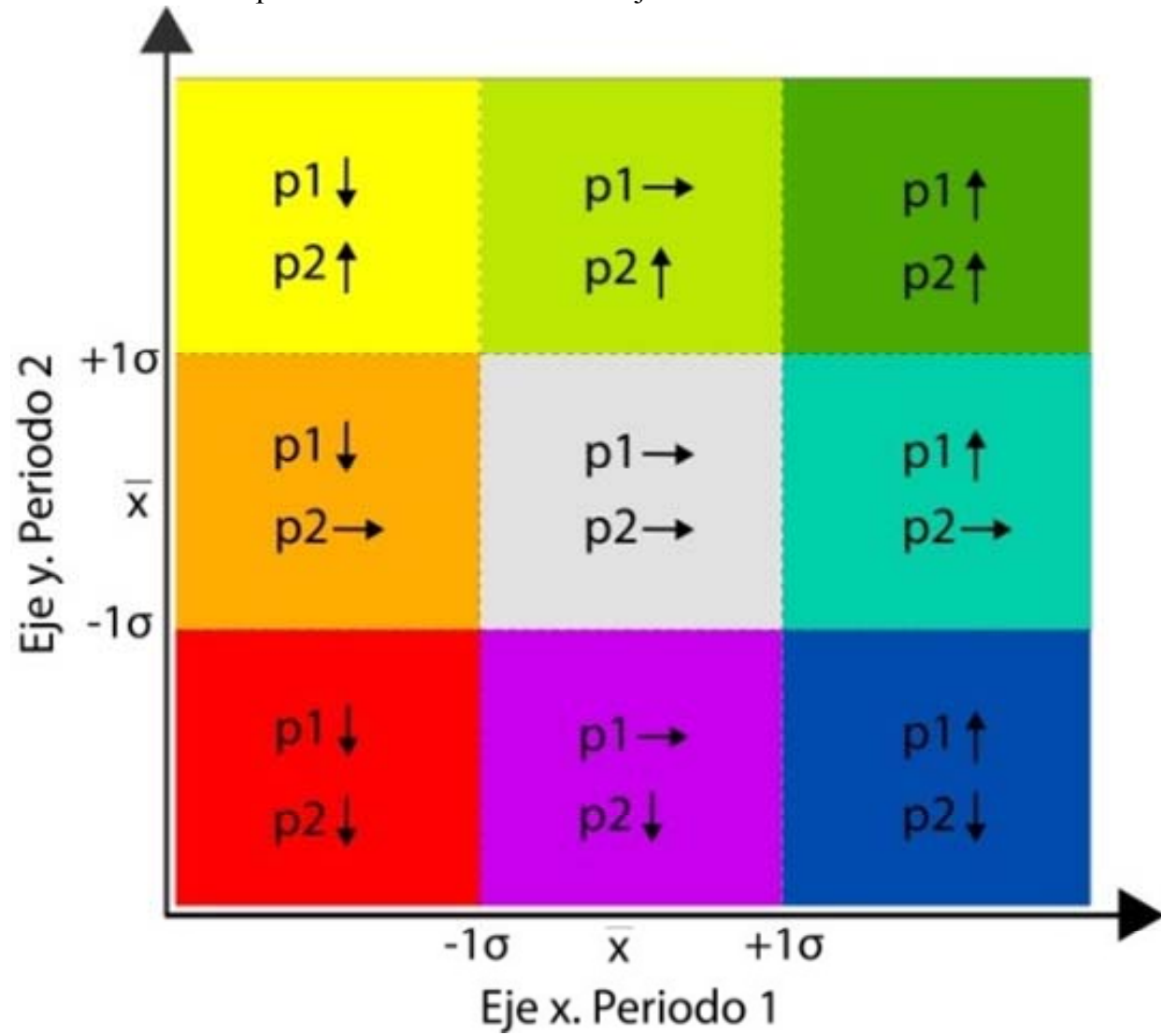

Fuente: Elaboración propia

Dónde:

$\uparrow$ es aumento de concentración

$\downarrow$ es disminución de concentración

$\rightarrow$ es variación de concentración dentro de la media

p1 es periodo 1

p2 es periodo 2 
En cuanto a la definición del ámbito de estudio, el área metropolitana de Sevilla, se ha partido de la delimitación de los espacios metropolitanos propuesta por Feria (2008) y actualizada según la información del censo 2011 (Feria y Martínez, 2016) en base a los principios de espacio de vida. El valor añadido que plantea la idea de trabajar en el espacio metropolitano sevillano es doble. Por un lado porque la llegada de inmigrantes se ha producido en este caso de una forma especialmente intensa en un periodo de tiempo muy corto lo que, como ya se ha comentado, permite reproducir el proceso de segregación prácticamente desde su inicio. Por otro lado, dicho sistema metropolitano se caracteriza por una estructura monocéntrica radiocéntrica, fundamentada en un centro metropolitano bien definido que funciona como motor de un desarrollo concéntrico, con núcleos interrelacionados que se pueden asociar a la regla rango/tamaño y cuya localización se va diluyendo en un gradiente de centro a periferia. Esto permite de estudiar, más allá de los cambios en la distribución espacial de la población nacida en el extranjero, posibles patrones de difusión radial en las concentraciones de inmigrantes a lo largo de todo el territorio metropolitano.

\section{Estrategia residenciales, desde la preferencia a la restricción residencial}

La población inmigrante residente en el área metropolitana sevillana es escasa en relación con otros espacios metropolitanos españoles (Iglesias Pascual, 2015). En 2003 la población nacida en el extranjero representaba el 2,67\% de la población metropolitana pasando en 2013 a suponer casi el 6\% de la población total. Con un crecimiento continuo a lo largo de todo el periodo, se observa una ligera aceleración del proceso migratorio a partir de 2007 con una divergencia clara entre los dos grupos establecidos. Cada uno de los dos colectivos ha mostrado dinámicas temporales diferenciadas en su incorporación a la sociedad sevillana. En este sentido si inicialmente los residentes europeos del grupo 1 formaban el colectivo más importante tanto en cifras absolutas como relativas, al final del periodo estudiado es el grupo 2 el que predomina y en particular los migrantes nacidos en América del Sur siendo los originarios de África y resto de Europa con unos efectivos inferiores a los de la Unión Europea, los que completan la lista. De este modo, es importante recordar que el grupo 1 significaba al inicio del periodo estudiado menos del $1 \%$ de la población total mientras el grupo 2 suponía el 1,7\%. Al final del ciclo estudiado, la presencia de población extranjera había aumentado en 3,28\%, explicándose este crecimiento en el incremento del $90 \%$ de colectivo más estigmatizado (Tabla 2 y Figura 4).

Tras este primer análisis introductorio de las dinámicas poblacionales de cada uno de los grupos establecidos, partiendo de los principios teóricos asociados a la regla rango/tamaño, en primer lugar se plantea el análisis de las estrategias residenciales de los dos grupos de estudio establecidos a partir del análisis de las pautas de localización territorial de sus poblaciones en relación con la distancia al centro 
neurálgico de la área metropolitana. En este caso: el centro histórico de Sevilla considerado como tal desde una perspectiva funcional (Figura 5).

Tabla 2. Caracterización de la población en el área metropolitana de Sevilla entre 2003 y 2013

\begin{tabular}{|c|c|c|c|c|c|c|c|c|}
\hline Ã̃ O & $\begin{array}{c}\text { Población } \\
\text { total }\end{array}$ & $\begin{array}{c}\text { Población } \\
\text { extranjera }\end{array}$ & Grupo 1 & Grupo 2 & $\begin{array}{c}\text { Población } \\
\text { extranjera }\end{array}$ & Grupo 1 & Grupo 2 \\
\hline $\mathbf{2 0 0 3}$ & 1.447 .512 & 38.589 & 13.921 & 24.668 & $2,67 \%$ & $0,96 \%$ & $1,7 \%$ \\
\hline $\mathbf{2 0 0 7}$ & 1.506 .285 & 61.400 & 17.415 & 43.985 & $4,08 \%$ & $1,16 \%$ & $2,92 \%$ \\
\hline $\mathbf{2 0 1 3}$ & 1.592 .909 & 94.805 & 22.107 & 72.698 & $5,95 \%$ & $1,39 \%$ & $4,56 \%$ \\
\hline
\end{tabular}

Fuente: Elaboración propia a partir del Padrón Municipal de Habitantes 2003, 2007 y 2013

Figura 4. Procedencia de la población nacido en el extranjero en el área metropolitana de Sevilla entre 2003 y 2013

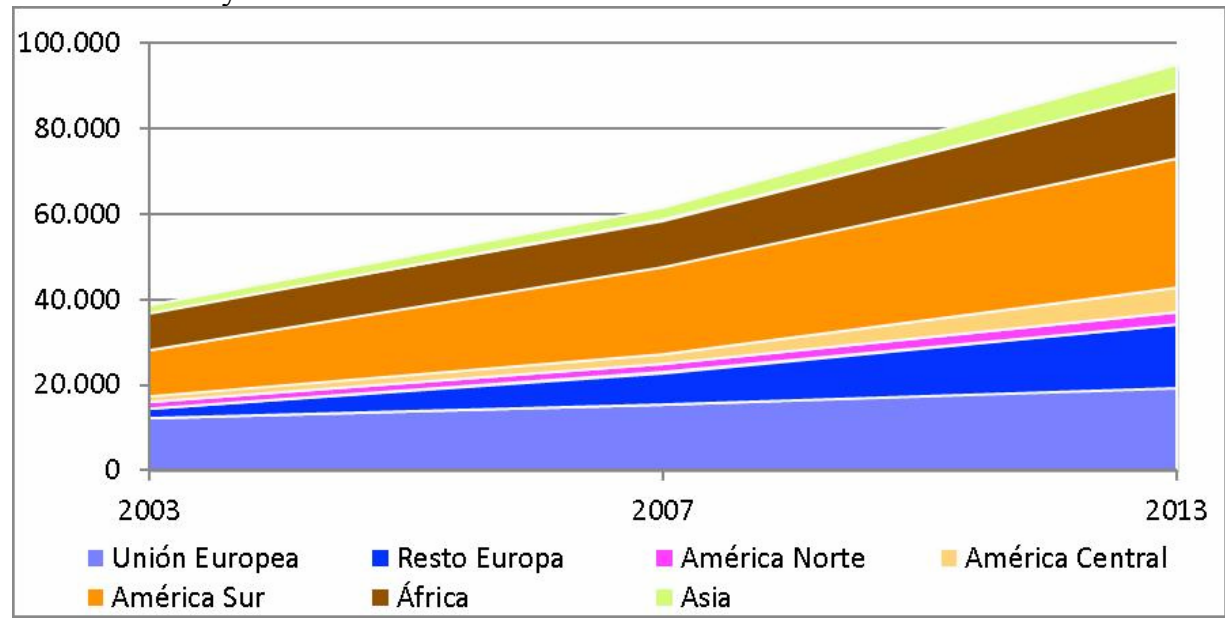

Fuente: Elaboración propia a partir del Padrón Municipal de Habitantes 2003, 2007 y 2013

Desde la escala metropolitana, se observa que las grandes tendencias de localización de la población nacida en el extranjero a lo largo del periodo analizado sigue un patrón en el cual los niveles de concentración van decreciendo desde el centro hacia la periferia de la área metropolitana llegando en todos los casos y a lo largo de los distintos periodos hacia los núcleos más alejados del centro. Por ello, se puede avanzar que ambos colectivos estudiados siguen de forma general pautas similares de localización a las de la sociedad de acogida. 
Figura 5. Distribución espacial de la población extranjera en la área metropolitana de Sevilla entre 2003 y 2013 (línea polinomial de nivel 4)

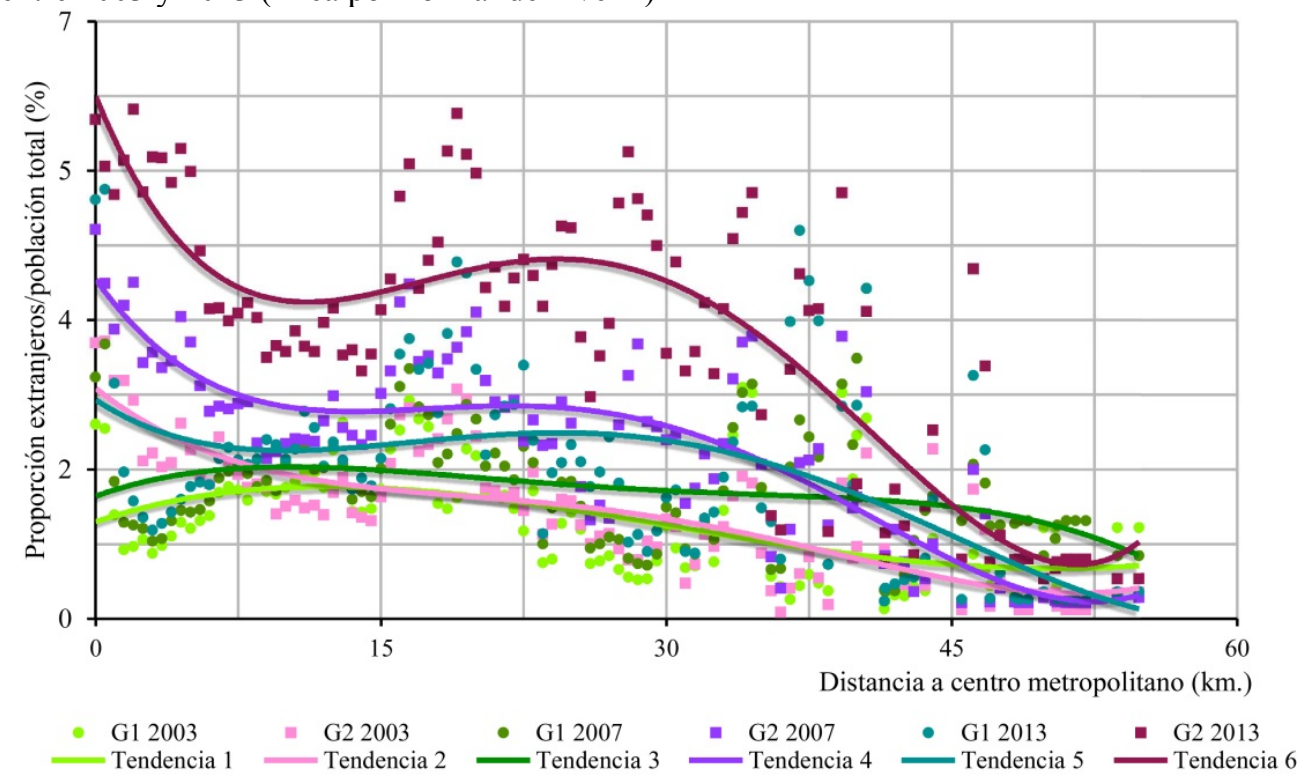

Fuente: Elaboración propia a partir de grid de población. Padrón Municipal de Habitantes 2003, 2007 y 2013 y MUCVA 2003, 2007 y actualización por elaboración propia a 2013

De este modo en 2003 los niveles de concentración son parecidos para los dos grupos, alrededor del $1,5 \%$ en la primera corona metropolitana, a unos 14/20 kilómetros del centro. En la segunda corona, entre los $20 \mathrm{~km}$ y $37 \mathrm{~km}$, el grupo 2 predomina ligeramente con niveles entre $1,4 \%$ y $0,8 \%$. A partir de esta distancia, el patrón territorial de organización de la población nacida en el extranjero cambia en los dos casos: el grupo 1, sigue manteniendo el grado de presencia mientras el grupo 2 tiende a desaparecer. En 2007 con el aumento de los efectivos del grupo 2 las pautas de localización en la aglomeración van variando: el grupo 1 resulta el más disperso en el territorio metropolitano mostrando una menor atracción por la ciudad central en sus estrategias residenciales. Atracción que sí se muestra con mayor intensidad en el grupo 2 que mantiene un patrón territorial con elevados niveles de concentración debido en parte al aumento progresivo de sus miembros. En 2013, a pesar de las grandes diferencias de concentración debidas al aumento continuo de la población del grupo 2, las pautas generales de distribución territorial en función de la distancia al centro mantienen sus tendencias. Para el grupo 2 el centro urbano del área metropolitana sigue siendo el espacio más atractivo, extendiendo su interés hacia el conjunto del espacio urbano del municipio sevillano y, de forma más tímida hacia los núcleos poblacionales a unos $15 / 30 \mathrm{~km}$ de la ciudad central. En contrate el grupo 1, muestra una variación en sus estrategias residenciales que se acercan a la del otro colectivo analizado, observándose una redistribución de la población hacia el centro 
histórico y hacia los núcleos urbanos situados a $20 \mathrm{~km}$ del centro, produciéndose una caída importante de la concentración de este colectivo en el resto del territorio.

Figura 6. Distribución de la localización residencial de los grupos de estudios en el área metropolitana de Sevilla, 2013.

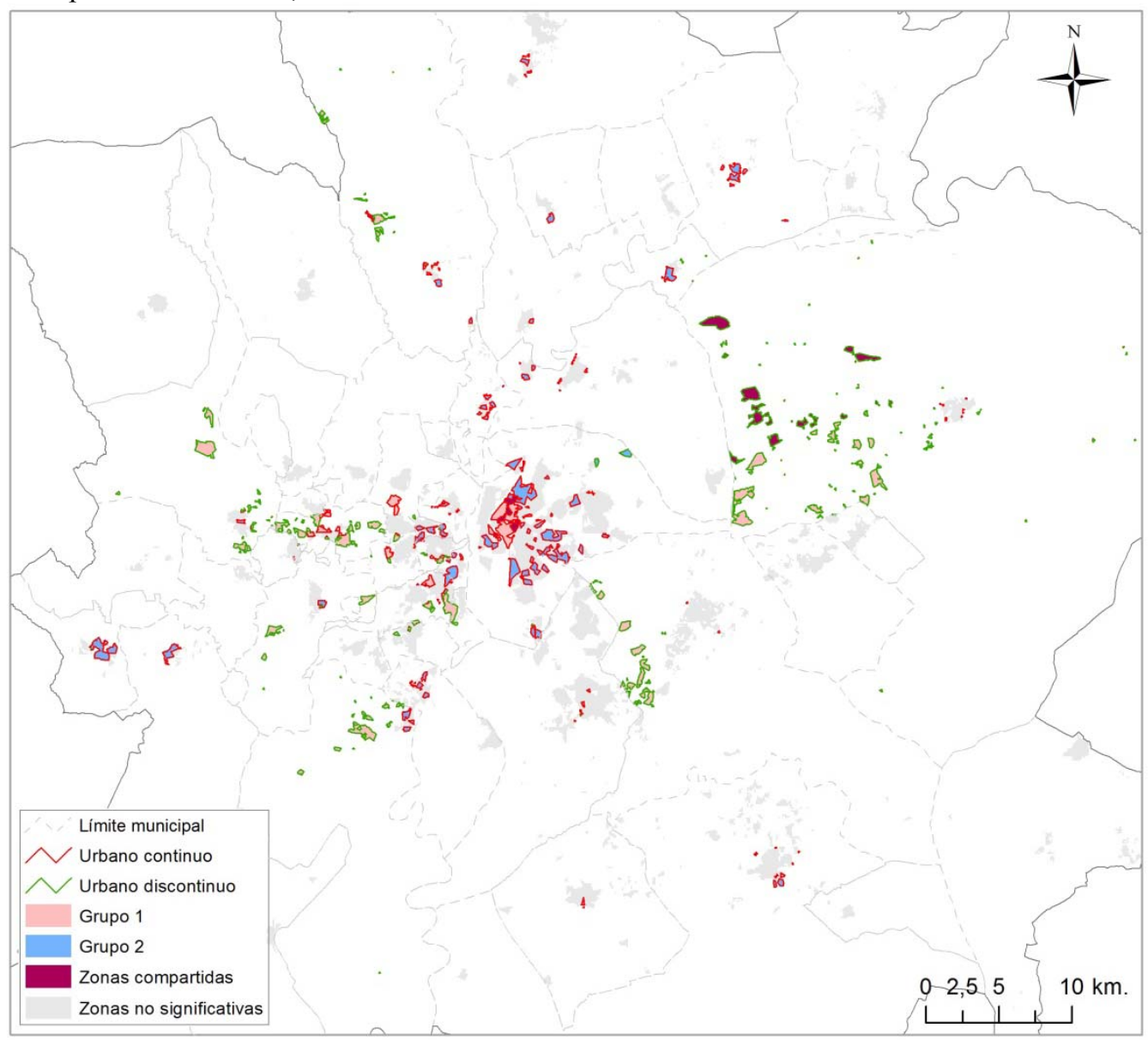

Fuente: Elaboración propia a partir de grid de población. Padrón Municipal de Habitantes 2013 y MUCVA actualizado según metodología a 2013

Partiendo de este análisis en relación al centro y la observación del comportamiento espacial de los dos grupos en 2013 (Figura 6), se observan pautas de localización residencial muy diferenciadas. Definiendo los espacios de mayor concentración como los territorios residenciales donde la densidad de población de cada grupo es superior a la media más una desviación típica, se observa cómo se mantiene el grado de disolución de la población del grupo 1 en el espacio metropolitano aunque su presencia en el casco histórico sevillano se hace más 
notable, muestra del proceso de gentifricación que se ha desarrollado recientemente en dicho espacio (Diaz Parra, 2012). Tal como se comentó anteriormente la observación más detenida de las características de localización de este primer colectivo, tanto en el centro histórico como en el resto del espacio metropolitano, permite afirma que en su mayoría repiten las pautas de la sociedad de acogida, buscando espacios de calidad residencial y medioambiental, compuesto de casas individuales adosadas o aisladas, de construcción reciente, con una buena accesibilidad a los grandes ejes de comunicación. Dichas pautas son las que explican de manera global los procesos de difusión espacial del tejido urbano en las áreas metropolitanas españolas y la de Sevilla más concretamente (De Oliveira Neves, 2010).

El grupo 2 tiende hacia una menor dispersión territorial a pesar de ser el más numeroso, ilustrando con ello un creciente proceso de concentración territorial. $\mathrm{Su}$ efectivos se localizan principalmente en los ámbitos periféricos de la capital andaluza, carácterizado por la edificación compacta, en barrios como La Bachilera, Poligono Norte Hermandades-La Carrasca, Los Pájaros o Amate, entre otros. La mayoría son barrios populares de bloques de pisos con una calidad habitacional media-baja, identificados en el imaginario social de la ciudad como ámbitos de conflictividad (Iglesias Pascual, 2016). Este modelo de distribución espacial del grupo 2, se repite en los municipios periféricos al oeste y al norte de la ciudad central, donde su localización suele coincidir con barrios de edificación compacta y escasas cualidades constructivas y ambientales. No obstante, a medida que los municipios se alejan del punto neurálgico del área, si bien es cierto que el número de residente del grupo 2 es reducido, el modelo residencial se va diversificando en el entramado urbano de diferentes núcleos rurales periféricos.

Por otro lado queda patente la escasez de ámbitos de concentración compartidos y por tanto de los espacios de convivencia de ambos colectivos. Son ámbitos puntuales en pequeñas unidades del norte y sur del casco historico. Fuera de este entorno, se localizan exclusivamente en urbanizaciones residenciales unifamiliares de baja o muy baja densidad, en núcleos secundarios sobre las terrazas occidentales del Guadalquivir y al este del espacio metropolitano.

A partir del análisis de la cartografía, se puede concluir que la preferencia residencial del grupo 1 se centra especialmente en el centro histórico y el espacio metropolitano más periférico, mientras que la restricción residencial del grupo 2 fundamentalmente se localiza en los barrios periféricos dentro de la ciudad central y aparecen en el espacio urbano compacto de la primera corona metropolitana. En este sentido resulta interesante relacionar cómo ha evolucionado en el tiempo los procesos de concentración residencial de ambos grupos para analizar si existe alguna relación con los procesos de segregación, rechazo y estigmatización territorial de algunos ámbitos metropolitanos (Tabla 3). 
Tabla 3. Concentración residencial de los grupos de estudio en el área metropolitana de Sevilla entre 2003 y 2013

\begin{tabular}{|c|c|c|c|c|c|c|c|c|c|}
\hline & \multicolumn{3}{|c|}{ Superficie total (ha.) } & \multicolumn{3}{|c|}{ Urbano continuo (ha.) } & \multicolumn{3}{|c|}{ Urbano discontinuo (ha.) } \\
\hline & 2003 & 2007 & 2013 & 2003 & 2007 & 2013 & 2003 & 2007 & 2013 \\
\hline Grupo 1 & 1.637 & 2.467 & 2.770 & 418 & 470 & 600 & 1.219 & 1.997 & 2.170 \\
\hline Grupo 2 & 1.682 & 1.819 & 1.730 & 966 & 1.546 & 1.685 & 716 & 273 & 45 \\
\hline Compartida & 1.518 & 800 & 708 & 225 & 192 & 135 & 1.293 & 608 & 573 \\
\hline
\end{tabular}

Fuente: Elaboración propia a partir de grid de población. Padrón Municipal de Habitantes 2003, 2007 y 2013 y MUCVA 2003, 2007 y actualización por elaboración propia a 2013

La distribución de la población en el territorio se traduce en particular por un cambio profundo en las pautas residenciales. Se observa como inicialmente, el grupo 1 privilegia los espacios discontinuos donde se encuentra el $94 \%$ de sus localizaciones. En realidad, el análisis comparado de la dinámica de localización de este colectivo en función de la morfología urbana pone de manifiesto un doble juego: por una parte crece la localización en espacios de baja densidad urbana y por otro decrece casi un 50\% el espacio compartido donde coexisten los dos grupos de estudio. Paralelamente se observa como el grupo 2, a pesar del incremento de su peso demográfico, apenas intensifica su presencia en el territorio, aumentando con ello la concentración en puntos muy concretos ya enclavados en el primer periodo. Esto muestra la tendencia de los miembros del grupo 1 a residir en zonas diferenciadas del grupo 2 y en gran medida visualiza el comportamiento que desarrolla la sociedad de acogida hacia los inmigrantes de menor renta y mayor rechazo social. Dicho proceso se confirma en 2013, donde se observa como los dos colectivos siguen aumentando sus efectivos de forma lineal, acentuándose el proceso de diferenciación en el espacio. Así el grupo 1 sigue expandiéndose en el territorio, manteniendo su densidad poblacional alrededor de unos 8 hab/has, a lo largo de todo el periodo. El grupo 2 por su parte, se retrae ligeramente en el territorio metropolitano acentuándose los procesos de concentración hasta llegar a un nivel medio de 42 hab./has: casi el doble que en 2007 y un $66 \%$ que al inicio del estudio. De nuevo, sus estrategias de localización hacen aumentar ligeramente los espacios densos de urbanización continua pero decrece su presencia en los espacios urbanos discontinuos, un $16 \%$ de lo que representaba en el periodo anterior y un 6\% de la superficie inicial. Por otro lado los espacios compartidos siguen bajando en los dos ámbitos morfológicos: un $30 \%$ de la superficie en los espacios urbanos continuos, a pesar del interés por el grupo 1 por esta morfología urbana y un $6 \%$ en las tramas discontinuas donde el colectivo del grupo 2 casi ha desaparecido.

De este modo resulta reseñable tanto la a concentración cada vez elevada del grupo 2 en zonas urbanas densas o continuas, como la desaparición progresiva de los 
espacios compartidos entre los dos colectivos. Ambos aspectos permiten mostrar cómo se ha producido el proceso de segregación espacial ocurrido a lo largo de los 10 años analizados, visualizando la existencia de estrategias de rechazo espacial y por tanto social por parte del grupo 1. Las preferencias residenciales del grupo 1, como ya se indicó, analizados como muestra del comportamiento de la sociedad de acogida, refleja la creciente segregación y concentración espacial del grupo 2 , y en general de los estratos sociales más depauperados, reflejando así la diferenciación social a través de la diferenciación espacial (Palh, 1975). De este modo al igual que el grupo 1 se ha tomado como muestra de la sociedad de acogida, el proceso de segregación del grupo 2 sirve como botón de muestra del creciente proceso de diferenciación y exclusión social que se encuentra en la base de la sociedad postfordista.

\section{Concentración y estigmatización territorial de los barrios inmigrantes}

Para analizar con mayor detalle cómo se ha producido el citado proceso de concentración de cada uno de los grupos de estudio en el periodo establecido se ha elaborado una cartografía, a partir de un gráfico de doble eje, que permite visualizar en el espacio dichas dinámicas, localizar y entender territorialmente el recorrido de cada uno de los colectivos y con ello, descifrar el proceso de reorganización territorial y confirmar la existencia de unas estrategias de rechazo social (Figuras 7, 8 y 9). En este sentido cabe destacar como el $70 \%$ de las unidades territoriales básicas estudiadas tienen una dinámica dentro de las normas de cada grupo. Fuera de ellas quedan representadas las unidades espaciales que han tenido una dinámica de concentración territorial anormal es decir por encima o debajo de la media más una desviación típica en el primer o segundo periodo. De este modo se pone de manifiesto las diferentes dinámicas de concentración de cada colectivo sobre la población total de la unidad así como, en el entendimiento de los casos más extremos, una aproximación a algunas estrategias territoriales que pueden explicar en parte el proceso de concentración espacial de cada grupo.

Así el grupo 1 suele localizarse en zonas urbanas de calidad, con una buena accesibilidad en el exterior de las grandes aglomeraciones urbanas, ostentando un nivel de concentración bajo y estable a lo largo de todo el estudio. A pesar de su dispersión territorial, la desviación típica es inferior a 1 en los dos periodos poniendo de manifiesto una distribución homogénea alrededor de la media. Por su parte el grupo 2 es cada vez más visible a lo largo del periodo estudiado por el aumento de los efectivos y por las estrategias residenciales que tienden hacia una concentración territorial en medio urbano denso, en particular en la ciudad de Sevilla.

Aunque la evolución media de concentración del grupo 2 en las unidades espaciales haya aumentado en ambos periodos, la desviación a dicha media en el primer periodo es de $1,33 \%$, subiendo a $1,66 \%$ en el segundo, mostrando por tanto procesos de segregación territorial continuos en el tiempo. De hecho, si al igual que en el grupo 1, el 30\% del territorio no responde al modelo dominante, el 5\% lo hace 
de manera contundente en proporciones muy superiores al anterior. El análisis de estos extremos permite entender las particularidades del desarrollo territorial del área metropolitana de Sevilla y poner el acento sobre los procesos de concentración del grupo 2 que en determinados casos lleva a la estigmatización territorial de los ámbitos donde residen.

Figura 7. Dinámica de concentración espacial de los grupos de estudio
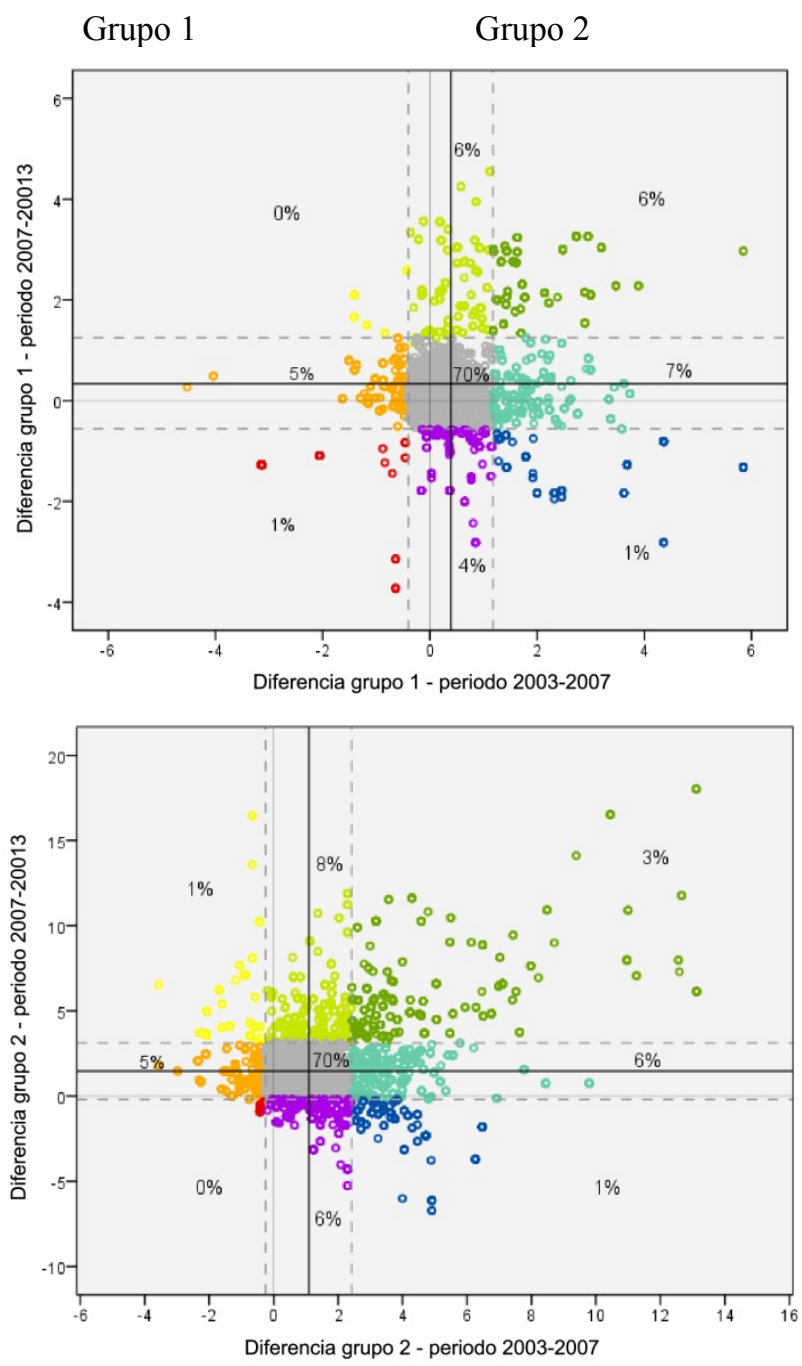

Fuente: Elaboración propia a partir de grid de población. Padrón Municipal de Habitantes 2003, 2007 y 2013 y MUCVA 2003, 2007 y actualización por elaboración propia a 2013 
Todo esto permite subrayar como en ambos colectivos existen espacios preferentes en los cuales han aumentado de forma importante las concentraciones durante todo el periodo de estudio. En el caso del grupo 1 estos ámbitos representan el 6\% del territorio, espacio en el cual el incremento de la concentración territorial ha sido bastante homogéneo. Dichos territorios se encuentra en el borde norte y Este del casco histórico de Sevilla, en espacios que han beneficiado de los grandes programas de renovación urbana originando con ellos los mayores procesos de gentrificación. A ellos se pueden añadir zonas de baja o muy baja densidad residencial distintos núcleos secundarios al oeste y este del área metropolitana.

Figura 8. Evolución espacial de la localización del grupo 1. Área metropolitana de Sevilla, 2003-2013

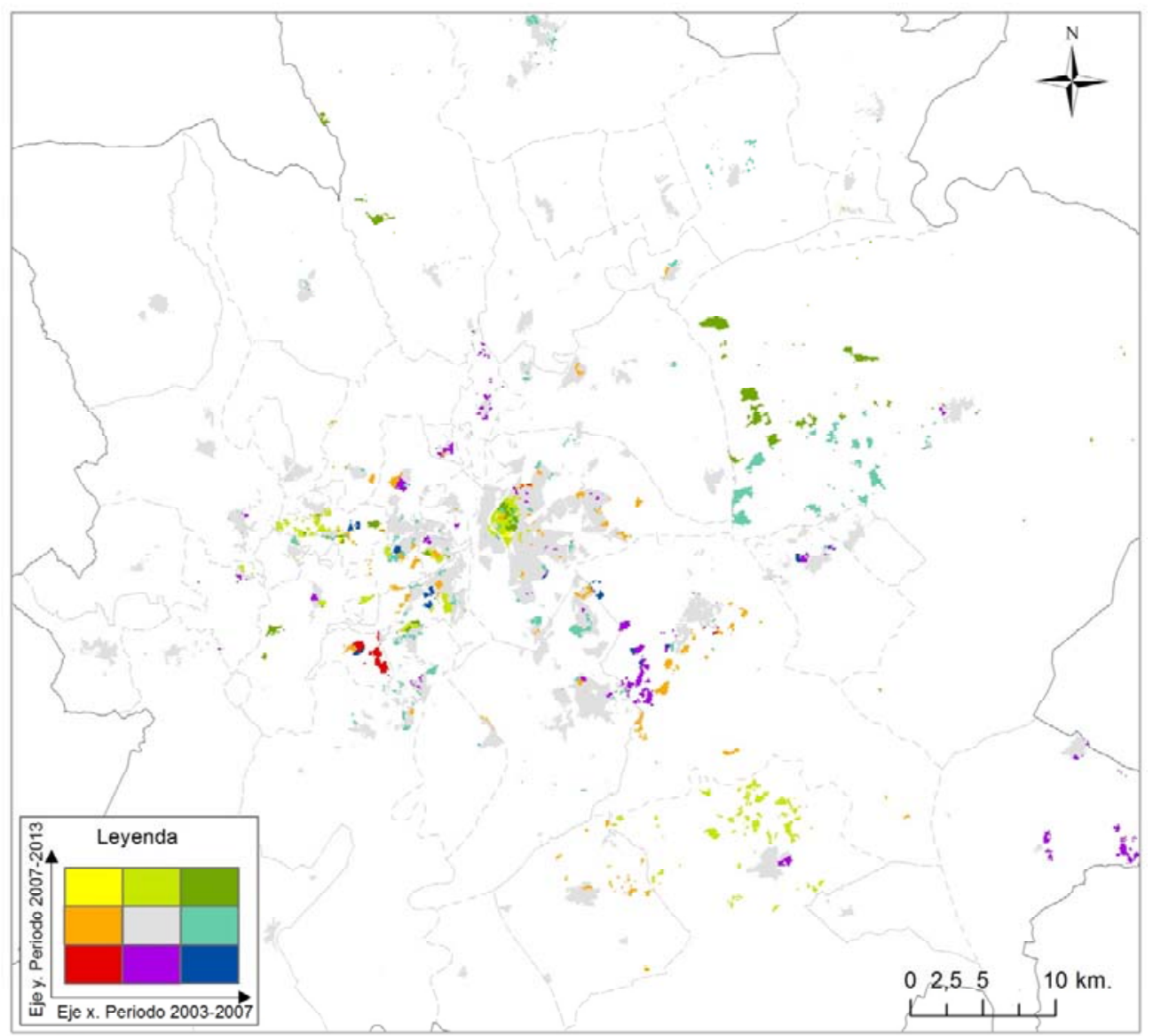

Fuente: Elaboración propia a partir de grid de población. Padrón Municipal de Habitantes 2003, 2007 y 2013 y MUCVA 2003, 2007 y actualización por elaboración propia a 2013 
Por su parte el grupo 2, desde el primer momento muestra una tendencia al incremento de la concentración de población a lo largo de los dos periodos analizados, siendo especialmente importante al inicio del periodo en los barrios del distrito Macarena, Polígono Norte, Los Pájaros y La Plata. Además de las diferencias en las estrategias residenciales y en las zonas de localización, se pueden observar diferencias en la intensidad del proceso de concentración: en el caso del grupo 2 el fenómeno de crecimiento continuado afecta a un 3\% del espacio de residencia (la mitad del grupo 1) pero con unos niveles muy superiores en los dos periodos.

Figura 9. Evolución espacial de la localización del grupo 2. Área metropolitana de Sevilla, 2003-2013

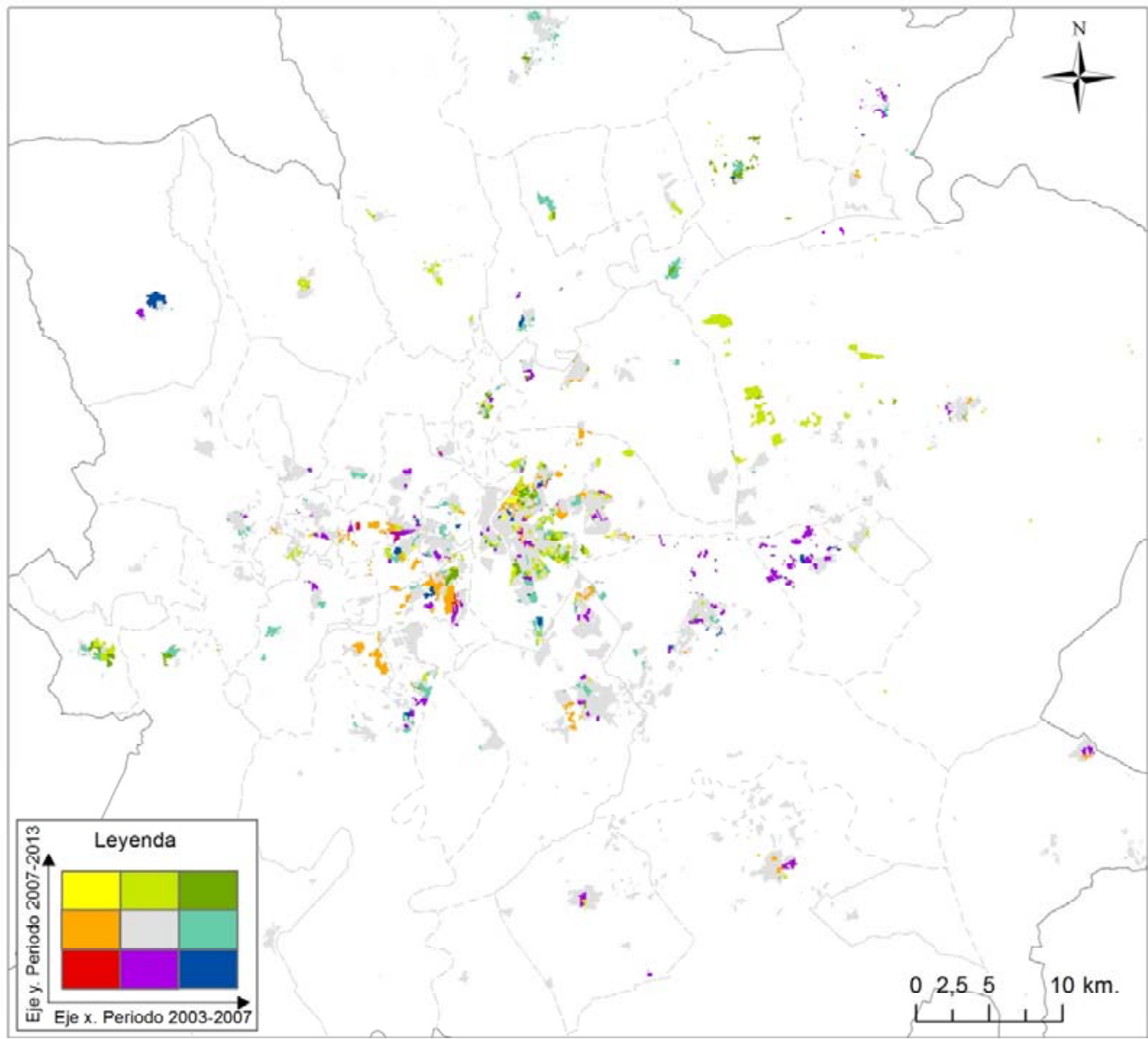

Fuente: Elaboración propia a partir de grid de población. Padrón Municipal de Habitantes 2003, 2007 y 2013 y MUCVA 2003, 2007 y actualización por elaboración propia a 2013 
Este aspecto confirma los análisis anteriores que muestran el proceso de concentración del grupo 2, con un carácter continuado y pausado a lo largo de todo el periodo estudiado, con niveles de crecimiento de la concentración hasta tres o cuatro veces más elevados que el grupo 1. Paralelamente la existencia de espacios que se desestiman como zona residencial en un periodo y se retoman en el siguiente reafirma la idea de que en el caso del grupo 2 no se puede hablar de propiamente elección o preferencia sino de restricción del mercado inmobiliario que dificulta su expansión a nuevos ámbitos residenciales. A su vez dicha restricción acentúa la concentración en ámbitos muy concretos lo que incide en su mayor visualización e identificación en el imaginario social metropolitano con barrios especialmente de la zona norte de la ciudad y por otro muestra como existen barreras para este colectivo a la hora de buscar nuevas zonas de residencia (Iglesias Pascual, 2016).

En este sentido se debe tener en cuenta que la mayor visibilidad social de la inmigración en la ciudad se asocia a fuertes proceso de concentración en ámbitos muy concretos de la misma. De este modo hay que tener en cuenta que la mera presencia de inmigrantes no estigmatiza un barrio. Para que se origine en el imaginario urbano un estereotipo que asocie el barrio con la inmigración deben darse diferentes circunstancias. En primer lugar albergar altos porcentaje de población extranjera o nacida en el extranjero, especialmente de aquellas nacionalidades que mayor rechazo levantan en la sociedad de acogida (Iglesias Pascual, 2016). A su vez esta nueva población acentúa su impacto en el imaginario social si su llegada se produce de manera continua y en un corto espacio de tiempo.

Teniendo en cuenta estos aspectos, en el caso de Sevilla solo en los ámbitos cercanos a la Macarena, Los Pajaritos y Su Eminencia se puede considerar que se ha generado un imaginario social que finalmente estigmatiza esos ámbitos como barrios de inmigrantes (Iglesias Pascual, 2014). Es en estos barrios donde los elevados porcentajes de concentración, superiores al $20 \%$, junto a las diferencias etnoculturales y la marcada precariedad de la situación económica ha generado el imaginario social que determina su estigmatización territorial como espacio poco deseables debido a la percepción de inseguridad y una hipertrofiada sensación de avalancha. Aunque no se pueda afirmar que todos los ámbitos metropolitanos donde se ha producido un aumento de la concentración del grupo 2 se puedan acabar desarrollando procesos de estigmatización territorial, sí resulta evidente que dichas zonas están visualizando ámbitos dónde es necesario aplicar políticas de integración intercultural o al menos desarrollar medidas preventivas ante la posibilidad de que se produzcan posibles déficits de convivencia.

\section{Conclusiones}

El desarrollo de la propuesta metodológica planteada en la investigación, basada en el análisis el comportamiento espacial de la población nacida en el extranjero, ha permitido poner en práctica un enfoque complementario al que normalmente se aplica 
en el estudio de la segregación y las preferencias residenciales de la sociedad de acogida y las diferentes nacionalidades. El hecho de separar la población de estudio en dos grupos según su nivel de rechazo social ha permitido, al extrapolar el comportamiento del grupo con menor o nulo rechazo (grupo 1) al de la sociedad de acogida, no solo analizar el comportamiento de ambos colectivos sino además realizar un acercamiento a cuál es el comportamiento residencial de la sociedad receptora y las tendencias a compartir los espacios de convivencia. En este sentido, al trabajar espacialmente no se ha pretendido entrar directamente sobre las motivaciones que inciden en dicho comportamiento pero ha permitido realizar un análisis sin la distorsión que se produce mediante el uso de encuestas. Por tanto resulta evidente que no se puede discernir claramente entre la tres grandes interpretaciones que explican las causas de la segregación pero ante la continua tendencia ambos grupos de estudio a concentrarse en zonas diferenciadas a medida que aumenta el porcentaje de inmigrantes económicos en el espacio metropolitano, se puede considerar que las causas de la segregación no se puedan explicar exclusivamente por desigualdades económicas y que haya que tener en cuenta tanto los prejuicios étnicos y la xenofobia como el rechazo del mercado residencial y de la sociedad de acogida para una visión global de la segregación. Esta necesidad de dar una interpretación global de la segregación deja ver la necesidad de incidir en el estudio de las percepciones y subjetividades sociales para un entendimiento holístico del proceso de segregación.

Por otro lado, la progresiva tendencia a la desaparición de los ámbitos de convivencia anteriormente referida, confirma la tendencia mostrada tanto en sociedades de acogida anglosajonas como europeas y la preferencia por barrios uniformes con el menor porcentaje de diversidad posible. Es decir el caso de Sevilla confirma la mayor tendencia a la autosegregación de los inmigrantes provenientes de países con alta renta e indirectamente de la sociedad de acogida. Del mismo modo las dificultades de la población del grupo 2 para buscar nuevas zonas de residencia inciden en la idea de su autosegregación espacial.

Por último resulta evidente que el efecto de la llegada de la población foránea y su concentración dentro Sevilla debe entenderse como una clara muestra de la validez de los estudios que se encuentran dentro de la teoría del rechazo. Tanto el comportamiento espacial como los estereotipos y la estigmatización territorial que se ha formado sobre los barrios de inmigrantes en la ciudad así lo muestran. A pesar de ello el escaso bagaje de convivencia con la inmigración no solo a Sevilla, si no en general a España dificultan poder analizar si la convivencia con el diferente, tal como sostiene la teoría del contacto, reduciría la aparición de conductas de rechazo y miedo. Por último hay que tener en cuenta que la progresiva concentración del grupo 2 en zonas muy concretas y la reducción de los espacios de convivencia, donde se producen concentraciones de ambos grupos, muestran como los estereotipos negativos que se asocian a las diferentes nacionalidades determinan en gran medida la preferencia del grupo $1 \mathrm{a}$ abandonar estos ámbitos y convivir en menor medida en aquellos ámbitos donde el grupo 2 es especialmente visible debido a su concentración. En general esta tendencia a la concentración y la segregación del inmigrante se puede considerar como una muestra más de los procesos de polarización social que se 
producen en el espacio urbano postfordista de manera creciente y que la dificultad de conseguir datos, al menos en el caso español, dificulta su análisis y visualización en el territorio.

\section{Bibliografía}

Allport, G. (1954): The Nature of Prejudice. Cambridge, MA: Addison Wesley.

Bader, M. y Krysan, M. (2015): Community attraction and avoidance in Chicago: What's race got to do with it? The Annals of the American Academy of Political and Social Science, 660 (1), 261-281.

Cea D'Ancona, M. A. (2009): La compleja detección del racismo y la xenofobia a través de encuesta: Un paso adelante en su medición. Revista Española de Investigaciones Sociológicas, 125, 13-46.

Cea D'ancona, M. Á. y Valles Martínez, M. S. (2015): Evolución del racismo y la xenofobia y otras formas conexas de intolerancia en España [Informe Encuesta 2014]. Madrid. OBERAXE. Ministerio de Trabajo e Inmigración.

Charles, C. Z. (2000): Neighborhood racial composition preferences: evidence from a multiethnic metropolis, Social Problems, 47, 379-407.

Charles, C. Z. (2001): Processes of racial residential integration. In A. O'Conner, C. Tilly, \& L. Bobo (Eds.), Urban Inequality: Evidence from four cities (217-271). New York: Rusell Sage Foundation.

Charles, C. Z. (2003): The Dynamics of Racial Residential Segregation. Annual Review of Sociology, 29, 167-207.

Clark, W. A. V. (2002): Ethnic preferences and ethnic perceptions in multi-ethnic settings. Urban Geography, 23, 237-56.

Clark, W. A. V. (2009): Changing residential preferences across income, education, and age: Findings from the multi-city study of urban inequality. Urban Affairs Review, 44 (3), 33455 .

Crowder, K. et al. (2006): Wealth, Race, and Inter-Neighborhood Migration. American Sociological Review 71(1), 72-94.

Crowder, K. y Krysan, M. (2016): Moving Beyond the Big Three: A Call for New Approaches to Studying Racial Residential Segregation. City \& Community, 15:1, 18-22. doi:10.1111/cico.12148.

De Souza Briggs, X. (Ed.) (2005). The geography of opportunity: Race and housing choice in metropolitan America. Washington, DC: Brookings Institution Press.

Dewaard, J. (2015): Beyond Group-threat: Temporal Dynamics of International Migration and Linkages to Anti-foreigner Sentiment. Journal of Ethnic and Migration Studies, 41 (7), 1041-1067.

De Oliveira Neves, G (1999): Análisis de la estructura social de Sevilla mediante la aplicación de la teledetección y de los sistemas de información geográfica, Sevilla, Junta de Andalucía. 
De Oliveira Neves, G., Hurtado Rodríguez, C. e Iglesias Pascual, R. (2016): Evolución en la localización residencial de la población nacida en el extranjero: el caso del área metropolitana de Sevilla (2003-2013). En Domínguez Mujica, J. y Díaz Hernández, R. (Eds.), Actas XV Congreso de la población española. Población y territorio en la encrucijada de las ciencias sociales.

Díaz Parra, R. I. (2012): Desplazamiento, acoso inmobiliario y espacio gentrificable en el caso de Sevilla. Encrucijadas. Revista Critica de Ciencias Sociales, 1 (2), 48-68.

Farley, R. et al. (1994): Stereotypes and Segregation: Neighborhoods in the Detroit Area. American Journal of Sociology, 100, 750-80.

Feria, J.M. (2008): Un ensayo metodológico de definición de las áreas metropolitanas en España a partir de la variable residencia-trabajo. Investigaciones Geográficas, 46, 49-68.

Feria, J.M. y Martínez, L. (2016). La definición y delimitación del sistema metropolitano español. Permanencias y cambios entre 2001 y 2011. Ciudad y Territorio (en prensa).

Gallego, F.J. (2010): A population density grid of the European Union. Population \& Environment, 31, 460- 473.

Goerlich, F.J., Cantarino, I. (2013): Geodemografía: coberturas del suelo, sistemas de información geográfica y distribución de la población. Investigaciones Regionales, 25, 165-191.

González Enríquez, C. y Álvarez-Miranda, B. (2006): Inmigrantes en el barrio. Un estudio cualitativo de opinión pública. Madrid, Ministerio de Trabajo y Asuntos Sociales.

Havekes, E., Bader, M., Krysan, M. (2016): Realizing Racial and Ethnic Neighborhood Preferences? Exploring the Mismatches Between What People Want, Where They Search, and Where They Live. Population Research and Policy Review, 35 (1), 101-126.

Herranz de Rafael, G. et al. (2012): Xenofobia en Andalucía. Análisis en municipios y ciudades con alta densidad de inmigrantes. Sevilla, Fundación Pública Andaluza Centro de Estudios Andaluces, Consejería de la Presidencia e Igualdad, Junta de Andalucía.

Hurtado Rodríguez, C. y De Oliveira Neves, G. (2015): El Perfil Urbano Metropolitano Andaluz En Base A La Desagregación Territorial De La Información Demográfica. En XXIV Congreso De La Asociación De Geógrafos Españoles. Análisis Espacial y Representación Geográfica: Innovación y Aplicación (Congreso). Zaragoza, España.

Ibraimovic, T. y Masiero L. (2014): Do birds of a feather flock together? The impact of ethnic segregation preferences on neighbourhood choice. Urban Studies, 51(4), 693-711.

Ihlanfeldt, K. R. y Scafidi, B. (2002): Black self-segregation as a cause of housing segregation: Evidence from the multi-city study of urban inequality. Journal of Urban Economics, 51(2), 366-390.

Ihlanfeldt, K. R. y Scafidi, B. (2004): Whites' neighborhood racial preferences and neighborhood racial composition in the United States: Evidence from the Multi-City Study of Urban Inequality. Housing Studies, 19, 325-359.

Iglesias Pascual, R. (2014). Generadores del discurso sobre inmigración extranjera y su difusión en el ámbito metropolitano. En I Simposium Internacional EDiSO 2014. Estudios sobre Discurso y Sociedad.

http://www.edisoportal.org/simposium2014/resumenes2014/resumenes2014-seminario-4. 
Iglesias Pascual, R. (2015): Segregación residencial, concepciones espaciales subjetivas y xenofobia en Sevilla. Determinando el umbral para la intervención social. Cuadernos Geográficos, 54(1), 230-256.

Iglesias Pascual, R. (2016): Espacio inducido y territorialización del discurso. Determinando el impacto socio-territorial del imaginario social sobre la inmigración en el Área Metropolitana de Sevilla. Documents d'Anàlisi Geogràfica, 62 (2), 299-325.

Kaufmann, E.P. y Harris, G. (2015): White Flight or positive contact? Local diversity and attitudes to immigration in Britain. Comparative Political Studies, 48- 12, 1563-1590.

Krysan, M. (1998): Privacy and the expression of white racial attitudes: a comparison across three contexts. Public Opinion Quarterly, 62 (4), 506-544.

Krysan, M.et al. (2009): Does Race Matter in Neighborhood Preferences? Results from a Video Experiment. American Journal of Sociology 115(2), 527-59.

Newman, B.J: y Velez, Y. (2014): Group Size versus Change? Assessing Americans' Perception of Local Immigration. Political Research Quarterly, 67(2), 293-303.

Openshaw, S. (1984): The modifiable areal unit problem. Concepts and Techniques in Modern Geography. Norwich, Geobooks.

Pahl, R. (1975): Whose city?. Harmondsworth, UK: Penguin.

Pettigrew, T. F., Wagner, U. y Christ, O. (2010): Population Ratios and Prejudice: Modelling Both Contact and Threat Effects. Journal of Ethnic and Migration Studies, 36-4, 635-650.

Quillian, L. (2002): Why is black-white residential segregation so persistent? Evidence on three theories from migration data. Social Science Research 31:197-229.

Shapiro, J. R. y Neuberg, S. L. (2008): When do the stigmatized stigmatize? The ironic effects of being accountable to (perceived) majority group prejudice-expression norms. Journal of Personality and Social Psychology, 95, 877-898.

Semyonov, M., Glikman, A. y Krysan, M. (2007): Population Size, Perceived Threat, and Exclusion: A Multiple-Indicators Analysis of Attitudes toward Foreigners in Germany. Social Problems, 54-4, 434-453.

Van der Bracht, K., Coenen, A. y Van de Putte, B. (2015): The Not-in-My-Property Syndrome: The Occurrence of Ethnic Discrimination in the Rental Housing Market in Belgium. Journal of Ethnic and Migration Studies, 41-1, 158-175.

Van der Laan Bouma-Doff, W. (2007): Involuntary isolation: ethnic preferences and residential segregation, Journal of Urban Affairs, 29(3), 289-309.

Wu, S.S. et al. (2005): Population Estimation Methods in GIS and Remote Sensing: A Review. GIScience \& Remote Sensing, 42 (1), 80-96. 